\title{
LA PROPORCIONALIDAD INTERPRETATIVA Y LA PROPORCIONALIDAD CONSTITUCIONAL
}

\author{
CARlos Alberto Burgoa TOLEdo ${ }^{1}$
}

RESUMEN: La proporcionalidad interpretativa y la proporcionalidad constitucional son dos formas de resolver casos difíciles que en la práctica se confunden indebidamente, pues en tanto el primero es un ejercicio de interpretación y se enfoca a las normas que han de aplicarse a un caso concreto, el segundo es un ejercicio de argumentación y se utiliza para conocer si se justifica o no el límite impuesto a un derecho constitucional mediante una norma secundaria en virtud de otro derecho o interés público que requiere atención, motivo por el cual, si se confunden ambos ejercicios, el resultado emitido en sentencias puede no satisfacer los principios del sistema legal, siendo importante su conocimiento para una aplicación debida.

PAlABRAs ClAVE: ponderación, principios, derechos constitucionales, moneda común, discreción judicial, lenguaje, propositos, interpetacion argumentación.

ABSTRACT: Interpretative proportionality and constitutional proportionality are two ways to solve hard cases that are unduly mixed and confused in practice, since the first one is an interpretative tool aimed to the norms to be applied to a concrete case, whilst the former one is an argumentative tool used to confirm whether a limit imposed to a constitutional right thru a sub-constitutional law due to the need of another people's right or a public interest in attention is justified or not, whereby the outcome in lawsuits might not satisfy the principles of a legal system, that is why it becomes important the acknowledge and understanding of the exact difference between them in order to arrive to a duly application of each one.

KEYWORDS: weighing, balancing, principles, constitutional rights, common currency, judicial discretion, language, purposes, interpretation, argumentation.

Sumario I. Introducción. II. Proporcionalidad. III. Comentarios finales. IV. Bibliografía

1 Doctor en Derecho por la UNAM, profesor de la FES Acatlán < contacto@ burgoa-toledo.mx>. 


\section{INTRODUCCIÓN}

T éxico se ha distinguido en los últimos años por adoptar figuras y prácticas jurídicas provenientes de otros países con la finalidad de enriquecer el bagaje normativo, y así, abonar a un mejor desempeño en las tareas que corresponden a las autoridades del Estado, lo que a futuro promete un mejor resultado y eficacia de sus funciones, ya sea legislativa, ejecutiva o jurisdiccional. Quizá la época en la que nuestro país exportaba figuras jurídicas (verbigracia el juicio de amparo) ha quedado atrás, y en la globalización - ahora en riesgo - y exigencia de respuestas jurídicas frente al dinamismo económico, social, político y cultural, México ha tenido la necesidad de adaptarse a tal ritmo y ser competente — también en lo jurídico- con los demás países, principalmente aquellos de primer mundo.

En esa inquietud espontánea y el compromiso de adoptar figuras y prácticas jurídicas internacionales, comienzan a fraguarse diversos errores derivado en ocasiones de la mala o errada recepción de tales ideas, novedosas para el país, lo que conlleva no sólo a un resabio conceptual, pues recordemos que la impartición de justicia versa sobre realidades expuestas de las cuales discurre un menoscabo económico, privación de la libertad, afectación patrimonial, entre muchas otras aristas que presenta la práctica profesional y la vida real. Por lo que el coste de "aprender" y "dominar" dichas novedades jurídicas no es sólo un ensayo académico que puede corregirse en el aula, sino que trasciende en la vida práctica y da pauta a un caudal de consecuencias con resultados reales.

Ejemplo de esta asunción "ciega" — por así llamarla - tanto de autoridades como de particulares, la tenemos con los derechos humanos. A colación de la reforma constitucional de junio de 2011, en donde se da nuevo nombre al Capítulo I del Título I de la constitucional para dejar de ser "De las garantías individuales" y pasar a ser "De los derechos humanos y sus garantías", 
se volcó una ola de amparos, escritos de demanda y diversas promociones de particulares evocando siempre el respeto a los derechos humanos, e incluso nombrando tratados internacionales (hasta antes desdeñados), como si fuera una equivalencia de forzar a los fallos de los juzgadores y sinónimo de hacer lo que a cada quien plazca, al igual que algunos juzgadores negaron el amparo a diversas personas morales por decir que los derechos humanos son eran exclusivos de las personas físicas (derivado del nombre que adopta el Capítulo I aludido de la Constitución), inercia que no sólo se dio en la práctica, pues todas las tesis escolares hablaban de derechos humanos, se crearon diplomados y congresos e incluso llegó a decirse que existen derechos humanos en la tributación. Desafortunadamente, antes de pedir o hablar sobre el tema, se debe conocer en su totalidad, amén que la reforma constitucional no fue una reforma sustancial (el qué) sino meramente formal, ya que no se crearon más derechos ni se han dejado de crear derechos o reconocer algunos otros, más bien se robusteció el efecto garantista por cuanto al comportamiento que deben tener las autoridades (el cómo), a fin de obtener un equilibrio de facultades en respuesta a las exigencias internacionales (e.g. caso Radilla, altamente trillado). Asimismo, se sigue hablando en algunos foros de "garantías individuales" dejando de lado que también existen las "garantías sociales". Peor aún, se piensa en las garantías individuales como sinónimos de derechos cuando lo cierto es que las primeras están a cargo del Estado (por virtud de obligaciones o prohibiciones), en tanto que los derechos son del particular y se aseguran - garantizancon los deberes del Estados, desconociendo así la correlatividad entre derechos y garantías. ${ }^{2}$

2 "There is, then, a familiar class of cases which can sensibly be talked about in terms of the 'correlation' of rights and duties, and it is tempting to suppose that whenever 'rights' and 'duties' or 'obligations' can be ascribed the pattern will recur. But while there are various implications between rights and duties, the pa- 
Por si eso no fuera poco, algunas personas siguen confundiendo las garantías individuales y sociales como aquellas que corresponde a una persona y a un grupo, cuando en verdad se relacionan con el tipo de conducta que corresponde al Estado - de "no hacer" y de "hacer", respectivamente - (las garantías de un grupo son garantías colectivas, más no sociales), a la par que sigue siendo motivo de confusión hablar de "interés legítimo" e "interés jurídico", visible no sólo en los particulares sino igualmente en las autoridades, entre muchos otros casos más que demuestran confusión de los temas.

Quizá estos resabios conceptuales se han comenzado a eliminar con el avance de la práctica y manejo de tema, pero lamentablemente no es el único en donde existe confusión teórica y práctica, pues dicha problemática se enfrenta ahora al hablarse de la "proporcionalidad", conocida también como "ponderación", con el resultado de que ahora todo se resuelve mediante el test de proporcionalidad, como si fuera una constante de la práctica jurisdiccional, sin atender a su verdadero sentido, ni a su relación con la discrecionalidad que ello implica ni la razonabilidad que le reviste. Y más aún, se le considera una forma de interpretación cuando en verdad se basa en la argumentación dejando atrás el sentido y significado de la norma, motivo por el cual, las siguientes líneas versan sobre la proporcionalidad, tanto en su sentido interpretativo como en su sentido constitucional, a fin de dar una noción breve, dada la extensión que merecen estas líneas, buscando abonar en el conocimiento de este tema actual.

ttern just sketched does not arise whenever rights and duties obtain. There are complications I cannot deal with here. I have sketched a notion of 'conceptual correlativity' restricted to rights held 'against' and duties or obligations 'owed' to specific persons. These do not exhaust the classes of rights and obligations: such restrictions need explaining and justifying. The notion is also restricted to 'passive' rights and 'active' obligations; and one might wonder whether some 'active' rights do not also correlate with obligations." Lyons, David, The correlativity of rights and duties, in 'Novus', IV, 1970, pp. 47-48. 


\section{Proporcionalidad}

En lo subsecuente, utilizaré la palabra "proporcionalidad" por ser la que mejor se adapta al test que lleva dicho nombre, así como a la esencia del ejercicio que se realiza. No utilizaré la palabra "ponderación" ya que ésta no es exclusiva del test de proporcionalidad, pues discurre igualmente para el "balance", lo cual, como ejercicios distintos, implican la confronta de valores inconmensurables o conmensurables. ${ }^{3}$

La proporcionalidad no es un tema exclusivo de alguna área del Derecho ni tiene un significado unívoco, por esa misma razón existe confusión en el tema. Se habla de proporcionalidad en lo penal cuando se trata del grado de las penas que se imponen a los delincuentes respecto del daño que han causado; asimismo, se habla de proporcionalidad tributaria cuando se refiere a la alícuota que deben pagar los contribuyentes respecto de los ingresos que perciben en determinado periodo de tiempo; de igual forma se habla de proporcionalidad cuando se trata de entender el mejor significado de la norma para sentar las bases de su interpretación ante la existencia de dos posibles soluciones; y también se habla de proporcionalidad cuando se busca confirmar si el límite impuesto a un derecho constitucional a través de una ley secundaria es acorde al fin que se busca. ${ }^{4}$ Estas dos últimas

3 Cabe señalar que dada la categorziación de los derechos en los EStados Unidos de Amércia, éste es el único país que no lleva a cabo el test de proporcionalidad, y se centra exclusivamente en el balance, lo que no acontecede con los demás países, en donde la falta de categorización, da lugar a realizer siempre el test de proporcionalidad, de lo que discurre afirmar que ambos tipos de ponderación (test de proporcionalidad y balance) son opuestos y no existe espacio para coexistir pues parten de valores distintos (conmensurables e inconmensurables).

4 "Proportionality is a central term in modern constitutional law. It serves different and various functions. Its meaning may change with the different roles it purports to fill. Thus, for example, the term proportionality as used in criminal law is not the same as the one used by administrative law and both terms differ from the term as 
formas de la proporcionalidad son el motivo de confusión común, pues se confunde a la proporcionalidad interpretativa con la proporcionalidad constitucional. Se dice indebidamente que el test de proporcionalidad es una herramienta de interpretación cuando lo cierto es que el test se basa en la argumentación. Es por eso que la proporcionalidad debe entenderse debidamente tanto para efectos interpretativos así como para efectos constitucionales, para lo cual, a continuación hablaré del primero.

\section{Proporcionalidad interpretativa}

Como primera acepción de la proporcionalidad, confundida altamente con la proporcionalidad constitucional, esta la proporcionalidad interpretativa. Se sabe que un texto de ley no siempre refleja en forma exacta y fiel lo que su autor quiso decir, de tal manera que existen diversos significados que pueden adjudicársele, y ante la existencia de opciones, debe elegirse aquélla que es correcta o más adecuada, y más aun, aquella que no sólo resuelva el problema presente, sino que igualmente sirva para casos similares a futuro, pues una norma no puede significar una cosa ahora y otra distinta después ya que se escribe para que perdure en el tiempo. Por eso, la interpretación debe apegarse en lo más posible a la intención del autor o a la intención del sistema legal, dependiendo el texto que se interprete. Y cuando existen dos o más posibilidades de interpretación, a fin de elegir la más adecuada para su aplicación, se lleva a cabo una ponderación. Por eso, esta primera

used by international law. Moreover, even with one legal field - such as constitutional law - the understanding of the term proportionality has changed over the years... Constitutional limitation clauses do not apply in every situation where two norms are in conflict with each other. Rather, they apply in those situations where one of the conflicting norms contains a constitutional right. The limitation clause and the notion of proportionality on which it is based were designed to provide both the justification for limiting a constitutional human right and the boundaries of such a justification." Barak, Aharon, Proportionality, constitutional rights and their limitations, UK, Cambridge University Press, 2012, pp. 146-147. 
acepción se llama proporcionalidad interpretativa, y se ubica en el contexto de la interpretación propositiva.

En principio, es sabido que cuando un texto normativo es claro, no queda más que aplicarlo (in claris non fit interpretatio); no obstante, si el texto normativo es oscuro, corresponde interpretarlo para entender su significado y sentido y así, aplicarlo; si el texto normativo es lagunoso, ambiguo o vago, corresponde integrarlo (mediante supletoriedad, llamado así en México), ${ }^{5}$ ya sea para aplicarlo directamente — si de la integración surge la claridad-, o a su vez interpretarlo para finalmente aplicarlo. De tal forma que cualquiera que sea la condición del texto normativo (claro, oscuro o lagunoso), siempre se busca aplicarlo - $\mathrm{O}$ cumplirlo, en el caso de los particulares-, por lo que la tarea que se busca es concreta (aplicación), independientemente de si para ello se requieren tareas abstractas (interpretación o integración), y las llamo tareas abstractas porque el producto de su realización no siempre se lleva al campo práctico o concreto, pues discurre en cualquier momento y ámbito, como el académico, por ejemplo, en donde lo interpretado por un alumno en una tesis de grado no se lleva a la práctica, además de no ser siempre correcto. Con lo anterior, es de señalar que la tarea abstracta de interpretación es propia de particulares y autoridades, pero la tarea concreta de aplicación es exclusiva de autoridades ya que trae aparejada la posibilidad de coacción, poder que no se confiere al particular pues éste, debe acudir siempre a la coacción del Estado si desea obtener algo de los demás particulares. ${ }^{6}$ Por tanto, el particular sólo cumple

5 Mientras que en España se le llama "subsidiariedad".

6 "La violencia del Estado se utiliza para apaciguar violencia que proviene de otros lados — con más o menos éxito, como se sabe, pero siempre en apoyo precisamente a dicha expectativa- Al concepto de violencia se le une una autorreferencia negativa y consecuentemente una paradoja: la violencia debe servir para hacer abortar la violencia. En el concepto de fuerza pública está incluida la exclusión de violencia. El concepto en cierra lo mismo violencia incluida que violencia excluida y es por consiguiente un concepto paradójico. Con el concepto de fuerza pública (o directamente traducido del alemán: violencia de Estado) se designa una solución de la paradoja. Esto conduce, en terminología actual, a la 
la norma más no la aplica. De allí el brocardo: "el desconocimiento de las leyes no exime su cumplimiento" sustantivado por el artículo 21 del Código Civil Federal.7 Así, el camino a la aplicación de la norma, puede mostrarse como sigue:

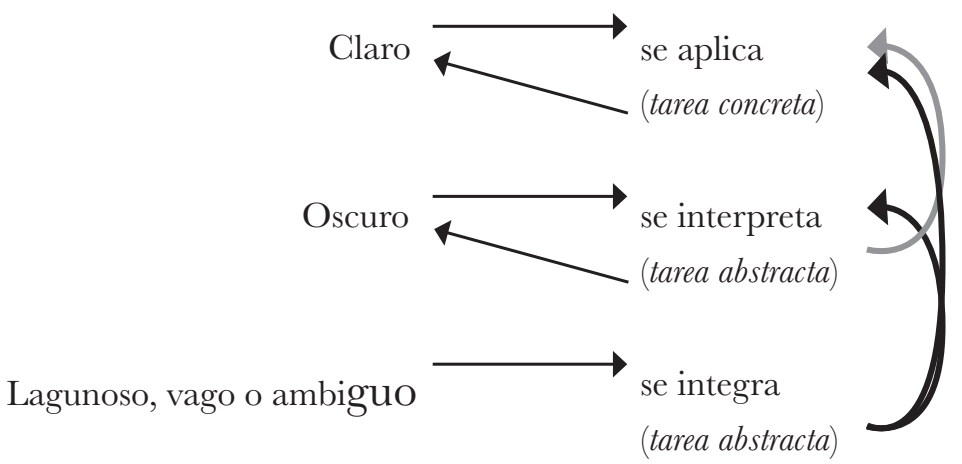

Figura 1. Texto normativo.

Dado lo cual, es de afirmar que el Derecho se escribe para aplicarse más no para contemplarse, de allí que cualquier ensayo sobre el mismo, debe ser apoyado en la realidad para arribar al derecho viviente (diritto vivente) y no sólo al derecho vigente (diritto vigente).

distinción violencia legítima/violencia ilegítima y postula la fuerza pública que su violencia es legítima. En esta medida la legitimidad pertenece a las características conceptuales de la violencia impuesta por el Estado - independientemente de cómo se le fundamente y de las controversias que surjan después-[...]. Si no hubiera violencia ilegitima latente (o virtual) que se opusiera, tampoco habría violencia legítima por parte del Estado.” Torres Nafarrate, Javier, Luhmann: la política como sistema, México, Ed. Fondo de Cultura Económica, 2004, pp. 213-214.

7 Artículo 21.- La ignorancia de las leyes no excusa su cumplimiento; pero los jueces teniendo en cuenta el notorio atraso intelectual de algunos individuos, su apartamiento de las vías de comunicación o su miserable situación económica, podrán, si está de acuerdo el Ministerio Público, eximirlos de las sanciones en que hubieren incurrido por la falta de cumplimiento de la ley que ignoraban, o de ser posible, concederles un plazo para que la cumplan; siempre que no se trate de leyes que afecten directamente al interés público. 
Así, la interpretación, cuando la norma es oscura, es de alta importancia previo a su aplicación, pues de no entenderse plenamente, el resultado puede ser devastador, por lo que la interpretación propositiva contempla tres elementos holísticos, los que abordaremos a continuació.

\section{A) Lenguaje}

Como cúmulo de palabras en un texto, la ley se base en un lenguaje, el cual es escrito, más en la tradición romano-canónica, del cual deriva en primer momento el significado, y posteriormente su sentido cuando se contrasta al texto con las políticas públicas que dan vida a la norma. Sin embargo, las palabras pueden tener una doble connotación, ya sea que formen parte del lenguaje privado o del lenguaje público. Esto es, que sean parte del hablar cotidiano y lo que en general se entiende de las palabras, o que sea parte del hablar propio y que en lo particular se entiende de las palabras. Lenguaje natural y lenguaje técnico sería una buena forma de demostrar este punto, en el lenguaje natural las palabras significan lo que se entiende en la vida cotidiana, mientras que en el lenguaje técnico las palabras significan algo específico para la disciplina o rama del conocimiento de que se trate. ${ }^{8}$

Ejemplo de esto es la palabra "obligaciones". Su significado natural implica una conducta que debe hacerse como deberes do-

8 "En primer lugar, tenemos al referente fáctico o empírico, que, de acuerdo con los positivistas lógicos, es aquel que puede señalarse con el dedo, ostensiblemente, o que se refiere a un 'estado de cosas' $[. .$.$] . Otro significado o referente de$ las palabras es el referente normativo. Es decir, hay palabras que se refieren a normas o, más bien, a modalidades normativas [...] Existe otro tipo de referente para determinar el significado de las palabras, el referente normativo. Hay palabras que no tiene un referente empírico ni un referente normativo, que no se refieren ni a normas ni a estados de cosas sino a estados de ánimo, es decir, a emociones o sentimientos." Berumen Campos, Arturo., Apuntes de Filosofía del Derecho, México, Cárdenas Editor Distribuidor, 2003, pp. 4-6, 10. 
mésticos en casa, tareas académicas, etc. Pero la misma palabra toma un significado distinto si la enfocamos en Derecho Mercantil cuando una sociedad emite obligaciones para allegarse de recursos sin incluir socios, las cuales se colocan a través del sistema financiero sin exceder el capital social, salvo que exista un proyecto que así lo justifique, dando lugar a que los obligacionistas tengan interés en el manejo de la sociedad sin contar con voto. La misma palabra no significa lo mismo en ambos casos. Por eso, la palabra no debe ser entendida sólo en su texto sino en su contexto. Pero el lenguaje no descansa sólo en natural o técnico, pues también se refiere a la cultura o lo que en una cierta región o zona significan las palabras (así como cuando se habla de "broma local"). Por ejemplo, si se dice a las persona que se realizará una manifestación en "los pinos", sin duda entenderemos que se trata a la residencia oficial del Presidente, pero si se realiza el mismo comentario a un extranjero que visita al país y no está habituado con nuestra cultura, pensará inmediatamente en un bosque, y la palabra no significa lo mismo para ambas personas. Por eso el lenguaje privado y lenguaje público no deben enfocarse sólo desde el lenguaje natural o el lenguaje técnico, sino también desde la cultura o costumbres de un lugar o sociedad que es lo que más representa el uso de las palabras y por ende, el significado y sentido que tienen. Sin embargo, pese a que el lenguaje pudiera en sí mismo resolver el tema interpretativo (lo que lo hace holístico), no es lo único que caracteriza a la interpretación propositiva, pues incardina también el propósito o intención.

\section{B) Propósito}

Las palabras no suelen expresar siempre la idea que se tiene al momento de escribirse, por lo que la confusión puede estancarse, por así decirlo, dentro del texto estático sino se le impregna el dinamismo de lo que se buscó. Ejemplo de esto lo tenemos con la extinta pena de muerte que contemplaba el artículo 22 Constitucional. El 
artículo hacía acreedor de la pena a diversos delitos, entre ellos, la "piratería", la cual no tiene igual connotación en el momento en que se escribió el texto magno respecto del día de hoy en que pudiera leerse. El texto magno se escribió cuando existía asalto a buques navíos y por tanto, era un mal que debía evitarse; muy distinto a lo que hoy en día significa dicha palabra, pues actualmente se refiere a la venta de mercancía que ha sido indebidamente copiada o producida en aras de confundir al comprador o, con el ánimo de obtener ganancias de una marca conocida cuyo modelo se emula. Cuando el lenguaje no basta para entender e interpretar la norma o el texto, se debe atender al propósito plasmado de forma implícita o explícita, ya sea el propósito (o intención) subjetivo del autor y el propósito (o intención) objetivo del sistema legal.

El propósito o intención subjetiva corresponde al autor del texto, ya sea las partes de un contrato, quien redacta un testamento o el cuerpo legislativo que crea una norma. En cualquier caso, tanto en el ámbito privado como en el ámbito público, existe un autor que busca un fin y plasma un documento con palabras que deben ser debidamente entendidas no sólo en su significado sino en su sentido - no sólo en forma sino en fondo-. Sin embargo, muy distinto es la intención escrita de la intención real. La intención escrita se refiere a la que se plasmada en palabras, mientras que la intención real es la que se busca y no siempre se plasma en palabras pues, en el caso de las normas, puede constar en su exposición de motivos, lo que confirma que la intención, para conocerse, no sólo cuenta con fuentes internas sino también con fuentes externas. Por ejemplo, las partes en un contrato acuerdan que cuando los servicios de telecomunicaciones se brinden en forma intermitente por fallas técnicas, la cantidad a cobrar será distinta respecto de las tarifas normales y, en caso de que se haya pagado ya el servicio, si la aclaración se realiza en los treinta días siguientes, corresponde la devolución de lo pagado en exceso más una cantidad adicional por "costo financiero", así llamado en contrato. Por tanto ¿qué tratamiento se debe dar a di- 
cho costo financiero? ¿se debe considerar indemnización por daños y perjuicios o se debe considerar intereses? Sin duda las personas buscarán inmediatamente en la norma en cuál de ellas encuadra cuando lo cierto es que primeramente se debe atender al texto del contrato para que, como lo dice el artículo 1851 del Código Civil Federal, ${ }^{9}$ se entienda con claridad qué quisieron decir y, entonces, ya con esa claridad, avanzar ahora sí al tratamiento que debe tener dicho pago adicional. No se trata de buscar la intención de un autor racional, sino la intención del autor tal cual se le conoce.

El propósito o intención objetiva corresponde al sistema legal, cuya inercia determina el significado real que corresponde a las palabras de un texto, contractual o normativo. La naturaleza de cada área del Derecho determina la interpretación que debe darse a las personas, la cual puede ser general, si aplica a todas las ramas del Derecho, o específica, si se refiere sólo a alguna rama del Derecho en particular. Intención general, por ejemplo, es la protección de los derechos humanos y de allí discurre el principio pro personae. Intención específica, por ejemplo, es la prohibición para la rama penal, la permisiva para la rama civil y mercantil, así como obligatoria para la rama fiscal, con lo cual se determina el mejor significado que corresponde dar a la norma para aplicarla. Un ejemplo de esto lo tenemos con el artículo 98 fracción III inciso c) de la Ley del Impuesto sobre la Renta, en donde se indica que las personas físicas, cualquiera que sea su nivel de ingresos, deben presentar declaración anual por su cuenta cuando en el año fiscal tuvieron dos o mas "empleadores" en forma simultánea. Por tanto, si el trabajador de una empresa a la par forma parte de una sociedad civil (como pudiera ser el caso de un maestro universitario), ¿deberá presentar la declaración anual de ISR? En la empresa es trabajador y cuen-

9 Artículo 1851.- Si los términos de un contrato son claros y no dejan duda sobre la intención de los contratantes, se estará al sentido literal de sus cláusulas.

Si las palabras parecieren contrarias a la intención evidente de los contratantes, prevalecerá ésta sobre aquéllas. 
ta con un patrón, pero en la sociedad civil no cuenta con patrón, entonces ¿a la palabra "empleador" de la ley se le debe identificar exclusivamente con la palabra "patrón” o debe dárseles la misma connotación aunque no signifiquen lo mismo? La respuesta es, debe dárseles la misma connotación pues la inercia de la rama tributaria es el pago de contribución, más no exentar o liberar de deberes a las personas. Por eso, en la práctica las autoridades hacendarias realizan invitaciones a las personas físicas, aunque cuenten sólo con un patrón y una fuente de ingresos distinta a la subordinación, pues ambos son empleadores, que no es lo mismo a patrón - hasta aquí el punto de lenguaje--, allende que es inercia o intención del sistema tributario que las personas presenten su declaraciones periódicas. En este peldaño se ubican cuatro grados de abstracción: i) acorde al autor del texto; ii) acorde al autor racional; iii) acorde al tipo de documento que se trate; y iv) acorde a los principios o valores del sistema normativo (general o específico). ${ }^{10}$

Es justamente en este punto en donde se da el ejercicio de ponderación, y toma relevancia la proporcionalidad interpretativa, pues si existen dos posibles interpretaciones que dar al texto o norma, se debe ponderar cuál es la más adecuada para aplicarse al caso. Por ejemplo, la fracción IV-Bis del artículo 127 de la Ley Federal del Trabajo dice que todos los trabajadores del establecimiento de una empresa mere-

10 "The fundamental problem in legal interpretation is the relationship between text and context, form and substance. Purposive interpretation views purpose as the context in whose light the text should be given meaning[...] First, purposive interpretation takes an integrative stance on the dichotomy between the intent of the author and the intent of the legal system [...] The second secondary question focuses on the intention of the text's author. It asks whether to focus on the 'true' intention or the 'expressed' intention[...] The third secondary question focuses on objective purpose. This purpose reveals the 'objective substance' of the text. It is the intention of the legal system, expressed in purposive presumptions that reflect the objective purpose of the system's various features and elements. The interpreter resolves internal conflicts among these presumptions by balancing them according to their relative significance." Barak, Aharon, Purposive interpretation, New York, Ed. Oxford, 2012, pp. 93-94. 
cen el pago de utilidades. Sin embargo, ¿qué sucede si se trata de una empresa que da servicios de subcontratación de personal a sus clientes?

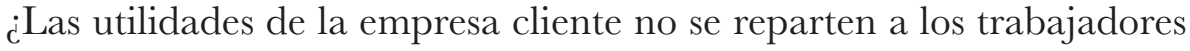
que ésta tiene en subcontratación pese a ser una unidad económica? o ¿sí deben repartirse a todos los trabajadores aunque legalmente no sean sus trabajadores? Negar la PTU a los trabajadores en subcontratación es actuar exclusivamente conforme a lo escrito (lenguaje), aunque no cumpla los brocardos de equidad y justicia de la rama laboral, y viceversa. Por tanto, el interprete debe ser consciente de que ambos significados son válidos y lo que corresponde ahora es determinar cuál es el sentido que se sentará para aplicarse al caso presente y ser precedente para futuros casos similares, pues es imposible pensar que lo decidido ahora no sea referencia para futuros casos similares pues no denotaría una constante en la tarea jurisdiccional, ${ }^{11}$ por lo que la elección del propósito ante dos posibles opciones, se resuelve con base en la discrecionalidad que constituye el siguiente paso como complemento de este.

11 "Temporal effects usually occur if decisions have detrimental effects on future generations - that is, if they have immediate benefits, although the costs will be felt years or decades later. The most prominent examples stem from the financial or environmental policy. A government can use debt to finance projects in order to gain additional votes. When the debts have to be paid back, the responsible politicians have often retired so that they are not accountable for the negative effects of their decisions. The situation is similar in the field of environmental policy. Emissions usually have long-term negative effects. The effects of measures aimed at the mitigation of climate change will only be felt in several decades, whereas the costs are already felt today. Many of the voters who today decide about the composition of the government may then not be alive anymore. At the same time, citizens who will feel the effect of environmental measures that are taken (or not taken) in 20 or 30 years often do not yet have a vote of might even not yet be born. Consequently, judicial review can protect future generations if courts correct short-sighted political measures. However, decisions in the field of public finance or the environment often require complex prognoses and specific expertise. Thus, courts might not be the appropriate actors to take an active role in the process." Petersen, Niels, Proportionality and Judicial activism, Fundamental rights adjudication in Canada, Germany and South Africa, New York, Cambridge University Press, 2017, pp. 31-32. 


\section{C) Discrecionalidad}

Dado que todas las normas, más aún en la tradición romano-canónica, no pueden prever en su totalidad los eventos futuros pues son escritas desde un presente que no conoce más allá de lo que ve, tienden a dejar en manos del juzgador la posibilidad de actuar, razonablemente, para dirimir tales controversias. Esto es a lo que se llama discrecionalidad, el poder de aplicar el criterio propio, dentro de los márgenes y principios que prevé el Derecho a los casos no previstos por ley. Quizá, el primer error en que se incurre cuando se piensa en la discrecionalidad es creer que es absoluta cuando lo cierto es que siempre es relativa, pues está acotada a los casos expresados por la norma, o incluso, de no existir un acotamiento expreso, aún así los principios y brocardos del sistema legal lo son, pues aún cuando en ciertos casos el juzgador pueda actuar sin sujeción estricta a la norma, los valores y principios siempre serán su límite y guía para tomar decisiones en los casos que así corresponde.

La discrecionalidad del juzgador opera en tres casos: i) respecto de hechos (qué valor darles mediante las pruebas que los acreditan); ii) respecto del sistema normativo (qué norma aplicar al caso concreto); o iii) respecto de la norma (en qué sentido aplicarla al caso concreto). ${ }^{12}$

El último caso es en donde se presenta la discrecionalidad para el efecto interpretativo. El juzgador cuenta con discrecionalidad, relativa desde luego, para dar el significado y sentido apegado a la intención del autor del texto o del sistema norma-

12 "I spoke of three objects of judicial discretion: fact, application of a norm, and the norm itself. The first type of discretion concerns facts vis-à-vis a norm; the second type deals with a norm vis-à-vis facts; the third involves the norm visà-vis itself and the rest of the normative system. The distinction among the three objects of judicial discretion is blurred. The difficulty is inherent in the fact that we do not have accurate instruments for determining what constitutes a fact and what a norm, and where the border between them lies." Barak, Aharon, Judicial discretion, Londres, Yale University Press, 1987, pp. 16-17. 
tivo, y así obtener el fin que busca el texto/norma. Sin embargo, si la norma establece límites férreos desde inicio, la discrecionalidad se ve reducida como sucede por ejemplo, con el primer párrafo del artículo 5 del Código Fiscal de la Federación. ${ }^{13}$ Asimismo, si no existe una remisión a la discrecionalidad del juzgador o autoridades, pero la norma se basa en principios, dado que éstos son de naturaleza abierta (open texture), nuevamente surge la discrecionalidad. Por tanto, sólo en los casos en que no se prevé aplicación estricta o interpretación restrictiva, y se trate de reglas y no de principios, el juzgador cuenta con discrecionalidad para que, razonable y justificadamente elija la mejor interpretación posible y, en caso de que existan dos interpretaciones posibles, el juzgador deberá realizar una "ponderación" dando lugar a la proporcionalidad interpretativa, lo que gráficamente se puede mostrar en la figura 2 .

Con todo lo dicho hasta ahora, es de entender que la interpretación propositiva tiene como materia de trabajo una norma que ha de aplicarse en un determinando momento, y de ahí que no exista posibilidad de hacer el ejercicio en el sentido constitucional (por ejemplo, si el cumplimiento de una sentencia en juicio contencioso administrativo puede tomar cuatro meses, respecto de la sentencia en amparo que debe tomar tres días, ello no es motivo de proporcionalidad constitucional ya que no existe roce de derechos, más bien se trata de entender el propósito que a cada uno corresponde. Para el ejemplo brindado, el cumplimiento de sentencia en juicio contencioso administrativo atiende a

13 Artículo 5o.- Las disposiciones fiscales que establezcan cargas a los particulares y las que señalan excepciones a las mismas, así como las que fijan las infracciones y sanciones, son de aplicación estricta. Se considera que establecen cargas a los particulares las normas que se refieren al sujeto, objeto, base, tasa o tarifa.

Las otras disposiciones fiscales se interpretarán aplicando cualquier método de interpretación jurídica. A falta de norma fiscal expresa, se aplicarán supletoriamente las disposiciones del derecho federal común cuando su aplicación no sea contraria a la naturaleza propia del derecho fiscal. 
esquemas de legalidad en donde se debate el cumplimiento de deberes por parte de la administración pública, mientras que en el juicio de amparo se alega la violación a derechos, lo que requiere mayor atención, y por tanto, la intención de ambos juicios es diversa, insisto, sin que exista una confronta de derechos o principios.

$$
\text { Lenguaje }\left\{\begin{array}{l}
\text { público } \\
\text { privado }
\end{array}\right.
$$

Holítsico

$$
\text { Propósito } \begin{cases}\text { subjetivo } & \left\{\begin{array}{l}
\text { expreso } \\
\text { real }
\end{array}\right. \\
\text { objetivo } & \left\{\begin{array}{l}
\text { individual } \\
\text { general }
\end{array}\right.\end{cases}
$$

Discrecionalidad

Figura 2. Proporcionalidad interpretativa. Fuente: Burgoa Toledo, Carlos Alberto, Argumentación para los agravios de defensa jurídica, México, Dofiscal Thomson Reuters, 2018, p. 284.

\section{Proporcionalidad constitucional}

A diferencia de la proporcionalidad interpretativa, la proporcionalidad constitucional tiene como finalidad saber si se justifica la limitante impuesta a un derecho constitucional (derecho humano, natural o fundamental) por medio de una norma secundaria, en virtud de la atención que requiere el derecho de otro u otros, o el interés público. Con lo cual, es claro que la proporcionalidad constitucional, como reflexión que se muestra argumentativamente, no 
es un ejercicio de interpretación, pues no está en duda el significado o sentido de la norma sino el límite a un derecho constitucional, lo cual no se justifica interpretando sino argumentando.

En este caso se realiza nuevamente una ponderación mediante cuatro pasos, subtest o subprincipios, todos ellos diapráxicos ${ }^{14}$ y no holísticos, por lo que a diferencia de la proporcionalidad interpretativa en la cual, cualquiera de sus elementos puede servir para resolver el significado y sentido de la norma puesta en duda, en la proporcionalidad constitucional deben seguirse forzosamente los cuatro pasos que en adelante se comentarán, siendo enfáticos en que la proporcionalidad constitucional surge por tres elementos:

- Un derecho constitucional;

- Una norma secundaria que limite dicho derecho constitucional;

- Por virtud del derecho de otro o de otros, o de un interés público legítimo.

Lo que mostrado con porcentajes burdos (por señalar números al azar sin que éstos sean importantes, sino la idea que se da), implica que el alcance original de un derecho, se reduzca por una norma secundaria dada la atención que requiere otro derecho o interés público:

14 "Il complemento (l'insieme-complemento) dell'insieme degli eventi diapraxici è l'insieme degli eventi non-diapraxici. Chiamo eventi non-diapraxici gli eventi la cui realizzazione non richiede la mediazione di un atto, ossia gli eventi i quali non avvenano (non si compiano) attraverso l'attuazione di un atto. Ecco cinque esempi di eventi non-diapraxici: (i)... (ii) la prescrizione di un diritto..." Conte, Amedeo Giovanni, "Pragmatica negativa" in P. di Lucia (a cura di), Assiomatica del normativo, Filosofia critica del diritto in Luigi Ferrajoli, LED, Milano, 2011, p. 70. 
Derecho constitucional

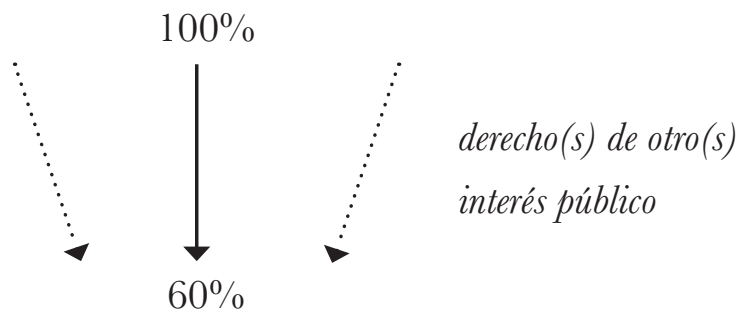

Norma secundaria

Figura 3.

Con el efecto predominante que se trata de limitación a derechos individuales (en donde el Estado está restringido a "no hacer"), más que a derechos sociales (de "hacer"). Por tanto, desde aquí se advierte la diferencia entre proporcionalidad interpretativa y proporcionalidad constitucional, pues mientras en el primer sólo esta puesto en duda una norma sin involucrar el derecho de otras personas o el interés público (es decir, se ve a la norma desde sí misma), en el segundo está puesto en duda el límite a un derecho constitucional, lo que implica que en cierta forma dos derechos se confrontan (es decir, se ve a la norma respecto de los demás), cuya solución se da con los siguientes pasos, para lo cual retomaré el esquema mostrado:

\section{A) Propósito legítimo}

Se refiere a que el derecho de otro u otros, o el interés público, sea legítimo, esto es, que se encuentre en forma implícita o explícita dentro de la Constitución. Por ejemplo, el derecho a un medio ambiente sano es explícito dentro del artículo 4 constitucional, mientras que el derecho al olvido, inmanente a los derechos ARCO, se encuentra implícito dentro del artículo 16 constitucional. De no 
ser legítimo dicho propósito, evidentemente la limitante al derecho constitucional que se ve afectado, no se justificará.

Pero no sólo lo implícito o explícito constituye el primer peldaño del test de proporcionalidad, pues más importante aún es el "nivel de urgencia" de la atención que requiere ese otro derecho (u otros derechos) o interés público. Esto es, que sea en verdad de tal magnitud que no pueda esperar. Por ejemplo, cuando en 2016 en la zona metropolitana se decretó la contingencia ambiental y por tanto, todos los automóviles dejarían de circular un día a la semana, independientemente de su placa o engomado, dado los altos niveles de contaminación como lo mostró la CAMe, era evidente la urgencia de tomar decisiones. Por eso, no basta con que se trate de un propósito que sea legítimo, pues además se requiere que dicho propósito tenga un nivel de urgencia que merezca atención inmediata.

Este primer paso, se refiere al derecho de otro u otros, o interés público (más no al derecho constitucional que es limitado, pues este es motivo de los siguientes dos pasos) el cual es marcado en negrillas y subrayado dentro del esquema antes mostrado para mejor referencia de este punto:

Derecho constitucional

$100 \%$

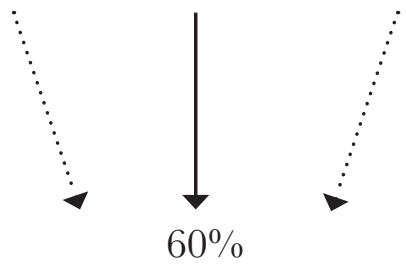

derecho(s) de otro(s) interés público

Norma secundaria

Figura 4. 


\section{B) Conexión racional (IDONeIDad)}

Se refiere a que el medio elegido para ser limitado, esto es, el derecho que sufrirá un detrimento en su alcance original, sea idóneo para hacer avanzar al derecho o derechos de otro, o interés público que se ha corroborado como legítimo y requiere atención. Así como burdamente se cuestiona de un medicamento para combatir el mal que se expone ante un médico y saber si en verdad servirá para curar el malestar que se comenta el médico, lo mismo sucede con este paso que incluye ambos elementos (derecho que se limita y derecho de otro u otros, o interés público), tan sencillo como eso. Sin embargo, no basta la idoneidad entre ambos elementos, sino que además, debe hacerse avanzar al derecho o interés público que requiere atención.

De no existir dicha idoneidad, no habrá justificación en la limitante al derecho afectado y por tanto, la medida tomada será inconstitucional.

\section{c) Necesidad}

Se refiere a que el medio elegido sea el que menos afecta a las personas y que aún así, avance al derecho o interés público necesitado. Nuevamente un ejemplo burdo podrá ayudar. Pensando otra vez en la salud, no es lo mismo la prescripción de pastillas a la prescripción de inyecciones intramusculares, pues evidentemente la primera opción no afecta al cuerpo como si puede suceder con el segundo. De tal forma que la afectación debe ser menor para los titulares de los derechos constitucionales que se eligen por el legislador o autoridades para limitarse, lo que se aboca exclusivamente al derecho limitado.

Sin embargo, puede suceder que una medida afecte menos a los titulares de los derechos pero la solución tome más tiempo, mientras que otra medida afecte más a las personas pero lleve 
menos tiempo. Volviendo al ejemplo burdo de la salud, es sabido que la prescripción de pastillas no son dolorosas pero tardan más tiempo en hacer efecto respecto de las inyecciones intramusculares (y que decir de las intravenosas). Dependiendo del fin que se busca, lo que se denomina "moneda común" (common currency), dará la decisión a tomar. Continuando con el ejemplo, si un paciente que sufre del estómago y desea recuperar pronto su salud para volver a sus actividades laborales, la "moneda común" es el "tiempo" y los principios en pugna son "salud" y "trabajo", por lo que el paciente seguramente elegirá las inyecciones intramusculares aunque sean dolorosas. Pero si las actividades laborales pueden esperar, entonces la confronta será distinta, pues el paciente ahora deberá decidir entre medicamentos de patente - de mayor precio - o medicamente genéricos - de menor precio-. En esta variable al ejemplo, la "moneda común" ahora es el "dinero" y los principios en pugna son "salud” y "economía”. Si el paciente decide favorecer su economía, el medicamento genérico será elegido, pero si decide favorecer su salud, el medicamento de patente será elegido.

Por tanto, la necesidad implica básicamente dos puntos:

i) Que existan alternativas de medios para dar avance al derecho o interés público que requiere atención; y

ii) Que el medio utilizado limite al derecho afectado en lo menor posible, es decir, no tomar el medio que esté a la mano, el primero que se piense o el que aparentemente es útil, sino el menos oneroso.

De tal forma que no basta con la existencia de alternativas sino que, además, se debe elegir aquella que cause la menor afectación posible. 
Como último paso del test de proporcionalidad, tenemos el equilibrio que debe existir entre el derecho afectado y el derecho de otro u otros o interés público, de tal forma que no exista una ganancia ni un detrimento en exceso, pues el punto es - precisamente - que exista proporcionalidad entre ambos, lo cual se puede resumir brevemente con el siguiente esquema, marcando una díada entre daño del derecho afectado y beneficio del derecho/interés que requiere atención para avanzar:

$$
\begin{aligned}
\geq & \text { (inaceptable) } \\
\text { Daño que sufre un derecho } & =\begin{array}{l}
(\text { aceptable }) \text { Beneficio al derecho(s) o } \\
\text { interés público }
\end{array} \\
\leq & (\text { aceptable })
\end{aligned}
$$

Figura 5. Equilibrio entre el derecho afectado y el derecho de otro u otros o interés público.

Como se muestra, si el daño es mayor al beneficio que se obtiene, el límite al derecho es inaceptable y por tanto inconstitucional. Si el beneficio es igual al beneficio obtenido, el limite es aceptable y por tanto constitucional. Si el daño que se sufre es menor al beneficio que se obtiene, el límite es aceptable y por tanto constitucional. Dado lo cual, los cuatro pasos referidos se pueden resumir de la siguiente manera: 
- Propósito legítimo $\left\{\begin{array}{l}\text { Fin legítimo }\left\{\begin{array}{l}\text { implícito } \\ \text { explícito }\end{array}\right. \\ \text { nivel de urgencia }\end{array}\right.$

- Idoneidad $\longrightarrow$ medios $-\operatorname{costos}^{-}$ (que ayude a avanzar a otro derecho/interés)

- Necesidad $\longrightarrow$ medida

- Proporcionalidad $\longrightarrow$ beneficio estricto sentido

Figura 6. Pasos del test de proporcionalidad. Fuente: Burgoa Toledo, Carlos Alberto, (coord.), El test de proporcionalidad, justificación en la limitación de los derechos, México, Thomson Reuters Dofiscal, 2017, p. 39.

Retomados del contexto internacional por la Primera Sala de la Corte en México (dado que no es un test que haya nacido en nuestro país, sino adoptado de la práctica alemana e inglesa), a través de los siguientes criterios emitidos:

- PRIMERA ETAPA DEL TEST DE PROPORGIONALIDAD. IDENTIFICACIÓN DE UNA FINALIDAD CONSTITUCIONALMENTE VÁLIDA. LOCAlizAción: [TA]; 10a. Época; 1a. Sala; Gaceta S.J.F.; Libro 36, Noviembre de 2016; Tomo II ; Pág. 902. la. GCLXV/2016 (10a.).

- SEgunda ETAPA Del test DE PROPORCiOnAlidAd. EXAMEN DE LA IDONEIDAD DE LA MEDIDA LEGISLATIVA. Localización: [TA]; 10a. Época; 1a. Sala; Gaceta S.J.F.; Libro 36, Noviembre de 2016; Tomo II; Pág. 911. 1a. CCLXVIII/2016 (10a.).

- TERCERA ETAPa DEL TEST DE PROPORCIONALIDAD. EXAMEN DE LA NEGESIDAD DE LA MEDIDA LEGISLATIVA. Localización: [TA]; 10a. Época; 1a. Sala; Gaceta S.J.F.; Libro 36, Noviembre de 2016; Tomo II; Pág. 914. 1a. CCLXX/2016 (10a.). 
- cuarta etapa del test de proporaionalidad. examen de LA PROPORCIONALIDAD EN SENTIDO ESTRICTO DE LA MEDIDA LEGiSlativa. Localización: [TA]; 10a. Época; 1a. Sala; Gaceta S.J.F.; Libro 36, Noviembre de 2016; Tomo II; Pág. 894. 1a. CCLXXII/2016 (10a.).

Lo cual dejaba en claro la postura de la Corte sobre el tema. No obstante, en febrero de este año, la Segunda Sala de la Corte emitió una jurisprudencia en la que inexplicablemente desdeña a la necesidad, como parte del test de proporcionalidad, para fines tributarios:

Época: Décima Época

Registro: 2016133

Instancia: Segunda Sala

Tipo de Tesis: Jurisprudencia

Fuente: Semanario Judicial de la Federación

Publicación: viernes 02 de febrero de 2018 10:04 h

Materia(s): (Constitucional)

Tesis: 2a./J. 11/2018 (10a.)

TEST DE PROPORCIONALIDAD DE LAS LEYES FISGALES. LA INTENSIDAD DE SU GONTROL GONSTITUCIONAL Y SU APLICACIÓN, REQUIEREN DE UN MÍNIMO DE JUSTIFICACIÓN DE LOS ELEMENTOS QUE LO CONFORMAN. El test de proporcionalidad es un procedimiento interpretativo para resolver conflictos de normas fundamentales, apoyado en los principios de igualdad e interdicción de la arbitrariedad o exceso, previstos en los artículos 1o., 14 y 16 de la Constitución Política de los Estados Unidos Mexicanos, y que requiere llevar a cabo, en primer lugar, un juicio de igualdad mediante la equiparación de supuestos de hecho que permitan verificar si existe o no un trato injustificado; en segundo lugar, el principio de proporcionalidad se conforma de tres criterios relativos a que la distinción legislativa: a) persiga una finalidad objetiva y constitucionalmente válida; b) resulte adecuada o racional, de manera que constituya un medio apto para conducir al fin u objetivo perseguido, existiendo una relación de instrumentalidad medio-fin; y, c) sea proporcional. Ahora, en materia tributaria la intensidad del escrutinio constitucional es flexible o laxo, en razón de que el legislador cuenta con libertad configurativa del sistema 
tributario sustantivo y adjetivo, de modo que para no vulnerar su libertad política, en campos como el mencionado, las posibilidades de injerencia del Juez constitucional son menores y, por ende, la intensidad de su control se limita a verificar que la intervención legislativa persiga una finalidad objetiva y constitucionalmente válida; la elección del medio para cumplir esa finalidad no conlleva exigir al legislador que dentro de los medios disponibles justifique cuál de todos ellos cumple en todos los grados (cuantitativo, cualitativo y de probabilidad) o niveles de intensidad (eficacia, rapidez, plenitud y seguridad), sino únicamente determinar si el medio elegido es idóneo, exigiéndose un mínimo de idoneidad y que exista correspondencia proporcional mínima entre el medio elegido y el fin buscado que justifique la intervención legislativa diferenciada entre los sujetos comparables. SEGUNDA SALA

Amparo en revisión 441/2015. Smart \& Final del Noroeste, S.A. de C.V. y otra. 5 de octubre de 2016. Cinco votos de los Ministros Eduardo Medina Mora I., Javier Laynez Potisek, José Fernando Franco González Salas, Margarita Beatriz Luna Ramos y Alberto Pérez Dayán; votó en contra de consideraciones Margarita Beatriz Luna Ramos. Ponente: Javier Laynez Potisek. Secretario: Jorge Jiménez Jiménez.

Amparo en revisión 845/2015. Tiendas Aurrerá, S. de R.L. de C.V. 5 de octubre de 2016. Cinco votos de los Ministros Eduardo Medina Mora I., Javier Laynez Potisek, José Fernando Franco González Salas, Margarita Beatriz Luna Ramos y Alberto Pérez Dayán; votó en contra de consideraciones Margarita Beatriz Luna Ramos. Ponente: Javier Laynez Potisek. Secretario: Jorge Jiménez Jiménez. Amparo en revisión 876/2015. Desarrollo Comercial Abarrotero, S.A. de C.V. y otras. 25 de enero de 2017. Cinco votos de los Ministros Alberto Pérez Dayán, Javier Laynez Potisek, José Fernando Franco González Salas, Margarita Beatriz Luna Ramos y Eduardo Medina Mora I.; votó en contra de consideraciones Margarita Beatriz Luna Ramos. Ponente: Javier Laynez Potisek. Secretario: Jorge Jiménez Jiménez.

Amparo en revisión 631/2017. José Armando del Toro Larios. 15 de noviembre de 2017. Mayoría de cuatro votos de los Ministros Javier Laynez Potisek, José Fernando Franco González Salas, Margarita Beatriz Luna Ramos y Eduardo Medina Mora I.; unanimidad 
de votos en relación con el criterio contenido en esta tesis; votó en contra de consideraciones Margarita Beatriz Luna Ramos. Disidente: Alberto Pérez Dayán. Ponente: José Fernando Franco González Salas. Secretario: Joel Isaac Rangel Agüeros.

Amparo en revisión 5 14/2017. Peñafiel Bebidas, S.A. de G.V. y otra. 29 de noviembre de 2017. Mayoría de cuatro votos de los Ministros Alberto Pérez Dayán, Javier Laynez Potisek, José Fernando Franco González Salas y Eduardo Medina Mora I. Disidente: Margarita Beatriz Luna Ramos. Ponente: José Fernando Franco González Salas. Secretario: Joel Isaac Rangel Agüeros.

Tesis de jurisprudencia 11/2018 (10a.). Aprobada por la Segunda Sala de este Alto Tribunal, en sesión privada del veinticuatro de enero de dos mil dieciocho.

Esta tesis se publicó el viernes 02 de febrero de 2018 a las 10:04 horas en el Semanario Judicial de la Federación y, por ende, se considera de aplicación obligatoria a partir del martes 06 de febrero de 2018, para los efectos previstos en el punto séptimo del Acuerdo General Plenario 19/2013.

Lo que contrasta evidentemente con los criterios sostenidos por la Primera Sala antes mencionados, cuya comparación es:

\section{Tabla 1.}

\begin{tabular}{ll}
\hline $\begin{array}{l}\text { Pasos del test de proporcionalidad según } \\
\text { la PRIMERA SALA }\end{array}$ & $\begin{array}{l}\text { Criterios del test de proporcionalidad se- } \\
\text { gún la SEGUNDA SALA }\end{array}$ \\
\hline Finalidad constitucionalmente válida & $\begin{array}{l}\text { Finalidad objetiva y constitucionalmente } \\
\text { válida }\end{array}$ \\
Idoneidad & $\begin{array}{l}\text { Finalidad adecuada o racional (relación } \\
\text { de instrumentalidad-medio) }\end{array}$ \\
Necesidad & - - - - - - \\
Proporcionalidad en sentido estricto & Proporcionalidad en estricto sentido \\
\hline
\end{tabular}

Amén que el subtest de necesidad es concebido como el corazón del test de proporcionalidad ("heart and soul") por las razones antes expuestas pues denota la menor afectación al derecho que se ve limitado, dado lo cual, se cuestiona ampliamente sobre el 
verdadero estudio que se ha hecho sobre el tema con la jurisprudencia citada ya que las teorías actuales y algunos fallos internacionales sólo han considerado reducir, a lo mucho, a la proporcionalidad en sentido estricto, más nunca desdeñar el subtest de necesidad. ${ }^{15}$ Así sucedió, por ejemplo, con la Corte Canadiense quien ha desarrollado esta opción en el caso $R \mathcal{F} R$-MacDonald $v$. Canada. Sin embargo, la jurisprudencia desdeña el test de necesidad y considera como subtest principal el de idoneidad, lo que resulta cuestionable respecto de la forma en que lo ha abordado el contexto internacional.

\section{iII. Comentarios finales}

Como puede advertirse, el test de proporcionalidad es un tema novedoso que debe aún madurar entre particulares y autoridades (administrativas y juzgadores) para arrojar un resultado adecuado que en verdad cumpla con este ejercicio, comenzado por primera vez en Alemania en 1958 y llegado a México cincuenta años después.

No es una tarea difícil pero si exigente, en tanto que demuestra el reto que se marca para nuestro país, en el cual participamos todos, y del cual se espera llegue al Estado Constitucional de Derecho que sus normas ostentan, para pasar de la letra a la práctica en beneficio de todos sus habitantes.

15 "The alternative proposed by Schlink is a reduced form of proportionality test, which only consists of the first three steps and does not include a balancing stage. However, this proposal has significant normative problems. If courts cannot resort to balancing, the less-restrictive-means test gains importance. And that has a structural effect on the later... The core of the less-restrictive-means test is the question whether a potentially less-restrictive alternative measure is as effective in achieving the public purpose as the reviewed measures. This estimation requires an empirical prognosis. In many cases, there will be uncertainty regarding the two measures that are compared." Petersen, Niels, op. cit., pp. 50-51. 
IV. BibLiografía

BARAK, Aharon, Fudicial discretion, Londres, Yale University Press, 1987.

—- Aharon, Proportionality, constitutional rights and their limitations, UK, Cambridge University Press, 2012.

—- Aharon, Purposive interpretation, New York, Ed. Oxford, 2012.

Berumen Campos, Arturo., Apuntes de Filosofia del Derecho, México, Cárdenas Editor Distribuidor, 2003.

Burgoa Toledo, Carlos Alberto, Argumentación para los agravios de defensa jurídica, México, Ed. Dofiscal Thomson Reuters, 2018.

—- Carlos Alberto, (coord.), El test de proporcionalidad, justificación en la limitación de los derechos, México, Ed. Thomson Reuters Dofiscal, 2017.

PaOlo di Lucia (a cura di), Assiomatica del normativo, Filosofia critica del diritto in Luigi Ferrajoli, LED, Milano, 2011.

Lyons, David, The correlativity of rights and duties, in "Novus", IV, 1970.

Petersen, Niels, Proportionality and Judicial activism, Fundamental rights adjudication in Canada, Germany and South Africa, New York, Cambridge University Press, 2017.

Torres Nafarrate, Javier, Luhmann: la política como sistema, México, Ed. Fondo de Cultura Económica, 2004. 